\title{
Flow channeling effect on a regenerator's thermal performance
}

\author{
Zei.-Chi. Chang* and Ping-Hei Chent \\ *Stone Industries Development Center, Hualien, Taiwan 970, Republic of China \\ tDept. of Mechanical Engineering, National Taiwan University, Taipei, Taiwan, \\ Republic of China
}

\section{Received 28 April 1997; revised 25 June 1997}

The aim of this study is to obtain a general formula from the measured heat transfer results in three regenerator matrices for evaluating the thermal performance of a regenerator matrix with various degrees of flow channeling. In this formula, a velocity ratio, $\phi$ and an area ratio, $\psi$ are determined from a nonuniform velocity profile at the regenerator exit. The nonuniform velocity distribution results from the flow channeling occurring at the contact surface between the regenerator matrix and the holding tube. A transient single-blow method was employed to measure the heat transfer rate in three inhomogeneous stacked-screen regenerator matrices. For a regenerator matrix with flow channeling, the thermal performance can be evaluated from the general formula and the measured radial velocity profile at the regenerator exit. The results show that the flow channeling effect may reduce the thermal performance of a regenerator matrix. (C) 1998 Elsevier Science Ltd. All rights reserved

Keywords: regenerator; flow channeling; heat transfer measurement

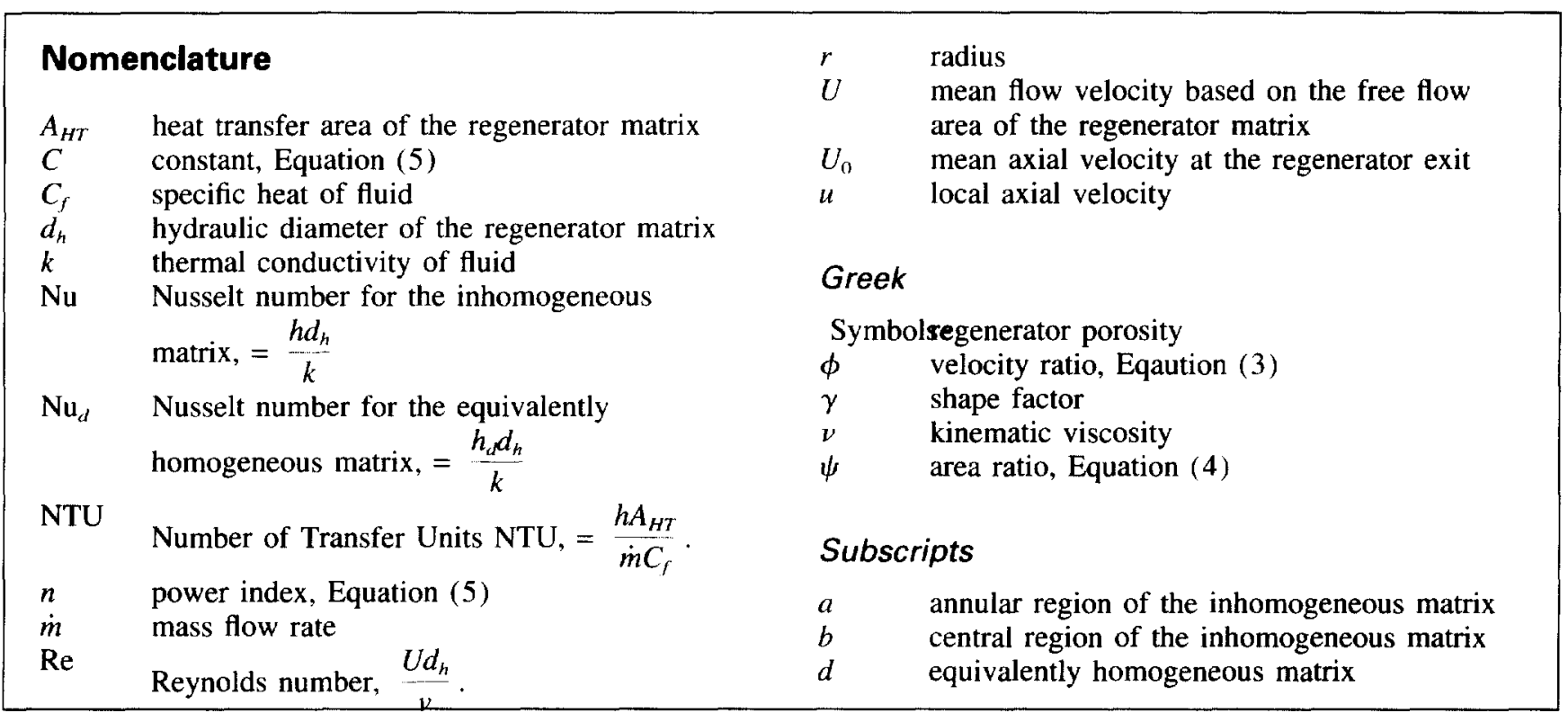

\section{Introduction}

In Stirling engines or cryocoolers, the regenerator matrix in which hundreds of wire screens are commonly stacked into an external tube, is one of the most crucial heat exchanger units for affecting the efficiency of the engine or cryocooler. To prevent flow leakage around the inner circumference of the tube, wire screens are usually made oversized. Thus, the wire screen oversize is defined as the difference between the diameter of the wire screen and that of the tube. Most engine or cryocooler designers have treated the fluid flow through the regenerator matrix to be uniform. However, some measured radial velocity distributions through a packed bed of spheres ' or through various regenerator matrices ${ }^{2}$ are not uniform. Jones ${ }^{2}$ attri- 
buted the flow inhomogeneity to the nonuniform porosity distribution in the regenerator matrix. It is expected that the flow uneven distribution through the regenerator matrix may affect the thermal performance of regenerators. Based on the measured velocity profiles at the exit of test regenerators, an analytical model proposed by Jones ${ }^{2}$ was used to assess the effect of flow inhomogeneity on the friction and thermal characteristics. Unfortunately, no measured heat transfer results on the regenerators were reported to justify his analytical model. An experimental study conducted by Chen et al. ${ }^{3}$ showed that the variation in the oversize value of a stacked wire-screen matrix with the external tube has an influence on the heat transfer rate between the through air flow and the wire-screen matrix. The heat transfer coefficients were measured for several regenerators in which the wire-screen matrix had the same dimensions but the holding tube had varying inner diameters. The measured results showed that the thermal performance of a regenerator matrix increases with a decrease in the inner diameter of the tube and this is attributed to the flow leakage around the contact surface between the regenerator matrix and the holding tube. However, the radial velocity distribution at the exit of the regenerator matrix was not measured.

This study aims to use the measured heat transfer results in three regenerators, with different radial velocity distributions, to verify the analytical model in Jones ${ }^{2}$ from which a general formula can be obtained for evaluating the thermal performance of a regenerator matrix with flow channeling. In this analytical model, a formula is derived to determine the ratio between the heat transfer rate in a perfectly homogeneous regenerator matrix and that in a regenerator matrix with flow channeling. Consequently, the heat transfer rate in any particular regenerator matrix can be calculated from the formula for a perfectly homogeneous matrix and the measured exit velocity distribution at the regenerator exit.

\section{Experimental Facility and Instrumentation}

This study uses a transient single-blow technique to measure the thermal performance of regenerators. Figures $l$ and 2 show the schematic view of the test regenerator and the experimental facility, respectively. Inlet and exit pressures and temperatures of the test regenerator matrix are measured using a pair of pressure transducers and thermocouples, respectively. Both the thermocouples are located on the centerline of the matrix. When the single-blow measurement is conducted, the inlet fluid flow temperature is raised sharply. After recording both inlet and exit temperature response curves, the heat transfer coefficient of the regenerator matrix can be determined by matching both predicted and measured exit fluid temperature response

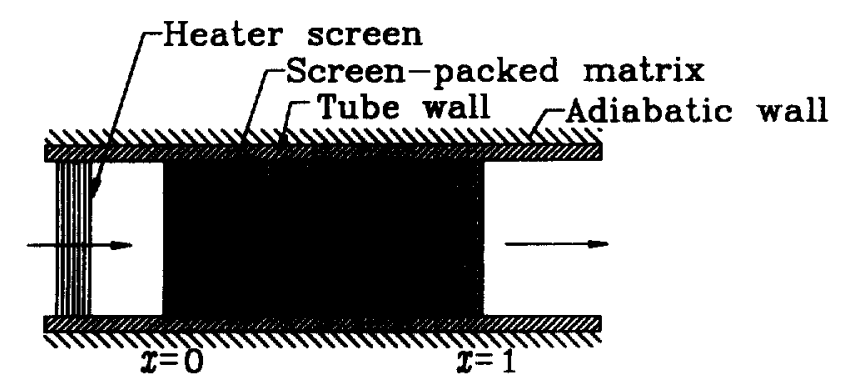

Figure 1 Schematic view of the test regenerator curves. The predicted exit fluid temperature histories can be obtained by solving the corresponding model for the single-blow system. A typical single-blow model contains two energy equations; one for the regenerator matrix and the other for the fluid flow (Liang and Yang ${ }^{4}$ ). The time period for recording the temperature history is 100 seconds and the sampling rate is $10 \mathrm{~Hz}$. Some assumptions are given in the single-blow model, including

1. one-dimensional and steady fluid flow,

2. constant material properties of wire screen and the tube wall,

3. negligible axial conduction in the fluid,

4. negligible conduction between wire-screen matrix and the tube wall,

5. negligible thermal capacity of the fluid,

6. adiabatic outer boundary of the tube wall, and

7. linear pressure gradient in the fluid flow through the wire screen matrix.

A detailed description of the single-blow technique and the experimental facilities can be found in Chen et al. ${ }^{3}$

When the transient single-blow technique is used, the predicted exit fluid temperature is compared to the measured temperature for determination of the heat transfer coefficient of the test heat exchanger. To obtain the fluid temperature response, the energy equations in the singleblow model are solved. In this study, an improved singleblow model ${ }^{5}$ was used for solving the fluid temperature history at the exit of the regenerator matrix. Compared to the model in Liang and $\mathrm{Yang}^{4}$, his improved model includes additional energy equations for the tube. Thus, the obtained heat transfer coefficients are higher in the present study than those in Chen et al. ${ }^{3}$ for the regenerator matrix with the same oversize value. This is because the single blow model in Chen et al. ${ }^{3}$ did not include the axial conduction of the test regenerator matrix.

In this study, the flow inhomogeneity through the regenerator matrix is also examined by measuring the radial velocity profile at the exit of regenerator. A hot wire probe and a TSI IFA-100 anemometer are used to measure the exit axial velocity profite. A linearly translated stage on which the hot-wire probe is installed is used to move the probe to the desired measuring locations. The distance between the hot wire and the test regenerator is kept at $2 \mathrm{~mm}$ while the measurements are conducted. The average velocity $U_{0}$ is then determined by integrating the measured velocity profile using the trapezoidal rule.

Three regenerators were tested and their dimensions and material properties are listed in Table 1. Exploying uncertainty analysis 6 , the estimated uncertainties for the Reynolds number, Re, the Number of Transfer Unit, NTU, the Nusselt number, $\mathrm{Nu}$, and the local axial velocity, $u$ are $4.3 \%, 2.2 \%, 5.7 \%$, and $2.4 \%$, respectively.

\section{Analytic Model}

In the analytical model in Jones 2, accounting for the effect of flow channeling on the thermal performance of the regenerator matrix, assumptions are given as follows:

1. The inhomogeneous wire screen matrix can be divided into two homogeneous submatrices arranged in parallel. One submatrix corresponds to the annular region through which the leakage flow occurs, and the other, 


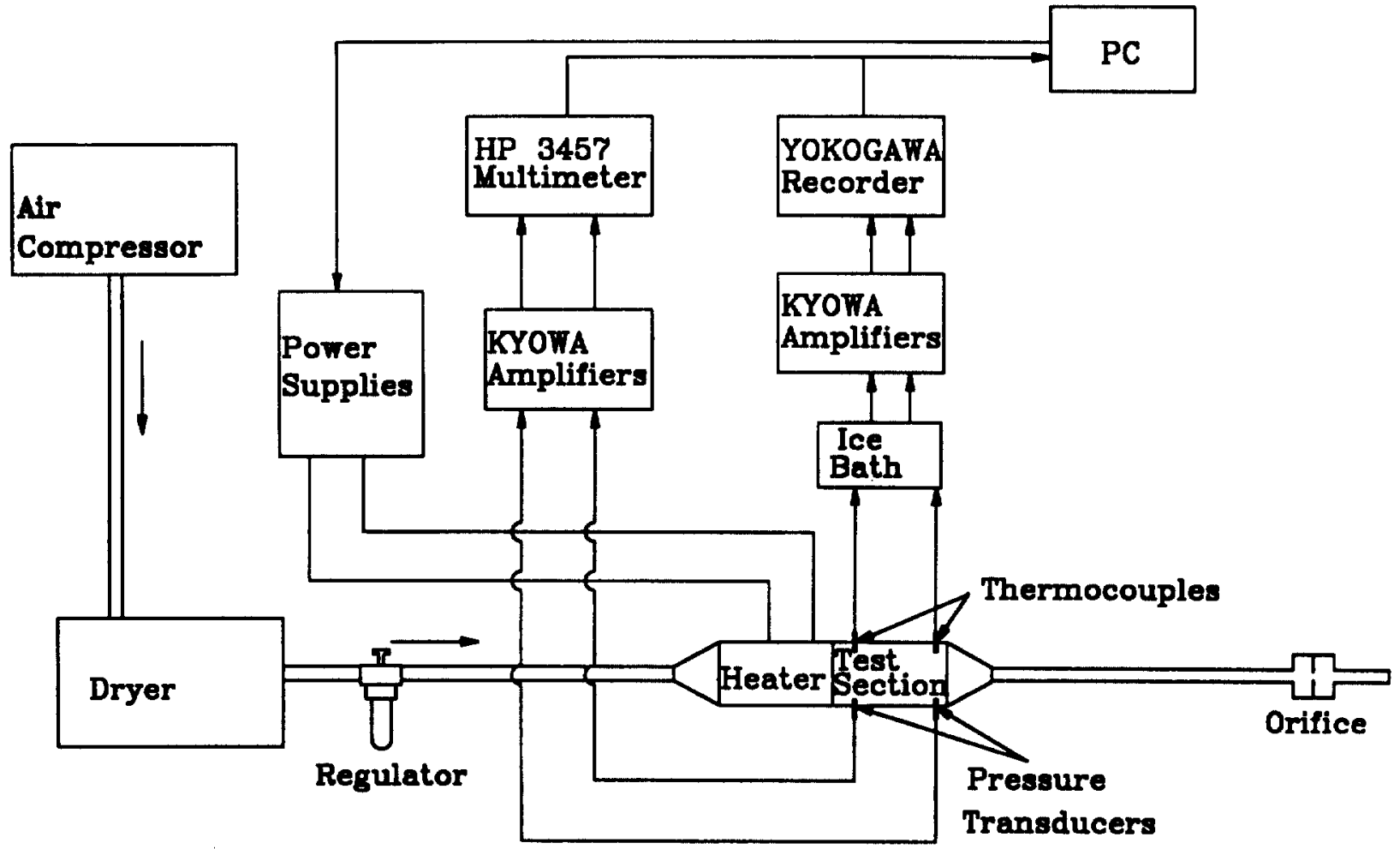

Figure 2 Schematic view of the experimental facility for single-blow measurements

Table 1 Geometric data and material properties of test regenerators

\begin{tabular}{|llll|}
\hline Regenerator Type & A & B & C \\
\hline Wire-Screen Material & SUS 316 & SUS 316 & SUS 316 \\
External Tube Material & SUS 316 & SUS 316 & SUS 316 \\
Length of Matrix, L (mm) & 60 & 60 & 60 \\
Inner Diameter of Wire-Screen & 19.0 & 18.7 & 18.5 \\
(mm) & & 19.05 & 19.05 \\
Diameter of Wire-Screen (mm) & 19.05 & 0.43 & 0.53 \\
Tube Thickness (mm) & 0.28 & 200 & 200 \\
Mesh Number of Wire-Screen & 200 & 560 & 560 \\
Number of Wire-Screen & 560 & 0.694 & 0.69 \\
$\epsilon$ & 0.704 & 0.12 & 0.11 \\
\hline$d_{h}(\mathrm{~mm})$ & 0.12 & & \\
\hline
\end{tabular}

the central region is assumed to be well-packed. The annual and central submatrices are denoted by subscripts $a$ and $b$, respectively.

2. These conceptual submatrices in assumption 1 are considered to have the same wire diameter, $d_{m}$ but different porosity, $\epsilon$.

3. The friction factors and heat transfer coefficients for each submatrix are constant over its length and cross section.

4. Entrance and exit losses are negligible.

5. The pressure across both submatrices is the same.

6. The measured exit flow velocity over each submatrix is uniform.

Two equations for the heat transfer coefficient ratio of the central and annular regions to that in a perfectly homogeneous matrix are given by

$\frac{h_{a}}{h_{d}}=\left(\frac{\epsilon_{d}}{\epsilon_{a}}\right)\left(\frac{1-\epsilon_{d}}{1-\epsilon_{c}}\right)^{n-1}\left(\frac{\phi}{(\phi-1) \psi+1}\right)^{n}$

$$
\frac{h_{b}}{h_{d}}=\left(\frac{1}{(\phi-1) \psi+1}\right)^{n}
$$

in which

$\phi=\frac{u_{a}}{u_{b}}$

and

$\psi=\frac{\pi r_{a}^{2}-\pi r_{b}^{2}}{\pi r_{a}^{2}}=1-r_{b}^{2}$

In Equations (3) and (4), $\phi$ is the ratio of the flow velocity through the annular region to that through the central region of the inhomogeneous matrix. $\psi$ denotes the ratio of the frontal area of the inhomogeneous matrix annular region to the total frontal area of the inhomogeneous matrix. $r_{b}$ denotes the outer radii of the central regions of 
the inhomogeneous matrix and the subscript $d$ denotes the equivalently homogeneous matrix.

The power index $n$ in Equations (1) and (2) is same as the measured heat transfer correlation between $\mathrm{Nu}$ and $\mathrm{Re}$ for test regenerators, given by

$\mathrm{Nu}=C \mathrm{Re}^{n}$

in which $C$ and $n$ are chosen to give the best fit with the measured data in the flow range of interest. The definitions of $\mathrm{Re}$ and $\mathrm{Nu}$ are expressed as

$\operatorname{Re}=\frac{U d_{h}}{v}$ and $\mathrm{Nu}=\frac{h d_{h}}{k}$

where $k$ is the thermal conductivity of the fluid. The hydraulic diameter of the inhomogeneous matrix, $d_{h}$, is defined as

$d_{h}=\left(4 \epsilon d_{m}\right) /[4 \gamma(1-\epsilon)]$

where $\epsilon$ denotes the porosity, $\gamma$ is a shape factor, and $d_{m}$ is the wire diameter of the wire screen. The value of $\gamma$ is 4 for wire screen. The detailed Equations (1) and (2), as well as $\epsilon_{a}$ and $\epsilon_{b}$ is described in Jones ${ }^{2}$.

Based on the definitions of $\mathrm{Re}$ and $\mathrm{Nu}$ and the formula in Equation (5), this heat transfer ratio between the submatrix of the inhomogeneous regenerator and the well-packed matrix can be expressed as:

$\frac{h_{a}}{h_{d}}=\left(\frac{\epsilon_{d}}{\epsilon_{d}}\right)\left(\frac{1-\epsilon_{d}}{1-\epsilon_{d}}\right)^{n-1}\left[\frac{\phi}{(\phi-1) \psi+1}\right]^{n}$,

$\frac{h_{b}}{h_{d}}=\left[\frac{1}{(\phi-1) \psi+1}\right]^{n}$

The ratio of the measured heat transfer coefficient in the inhomogeneous matrix to that in the homogeneous matrix is given by

$$
\frac{h}{h_{d}}=\psi \frac{h_{a}}{h_{d}}+(1-\psi) \frac{h_{b}}{h_{d}}
$$

where the $\psi$ value is determined from the measured radial velocity distribution at the exit of test regenerator matrix. By substituting Equations (1) and (2) into (9), the heat transfer coefficient of the perfectly homogeneous matrix can be evaluated from the measured one with various degrees of flow channeling through the test regenerator matrix. The value of $h / h_{d}$ is always less than 1 . When subject to the same mass flow rate, $h / h_{d} \leq 1$ indicates that the heat transfer in a homogeneous matrix is always greater than the heat transfer in an inhomogeneous matrix with flow channeling.

\section{Results and Discussion}

Figure 3 presents the radial velocity distributions at the exit of three test regenerator matrices. Similar velocity distribution through a stacked-screen matrix is reported in Jones 2. The maximum of the radial velocity distribution occurs near the tube wall. Regenerator $\mathrm{C}$ has the largest oversize and thus the velocity distribution for regenerator matrix $\mathrm{C}$

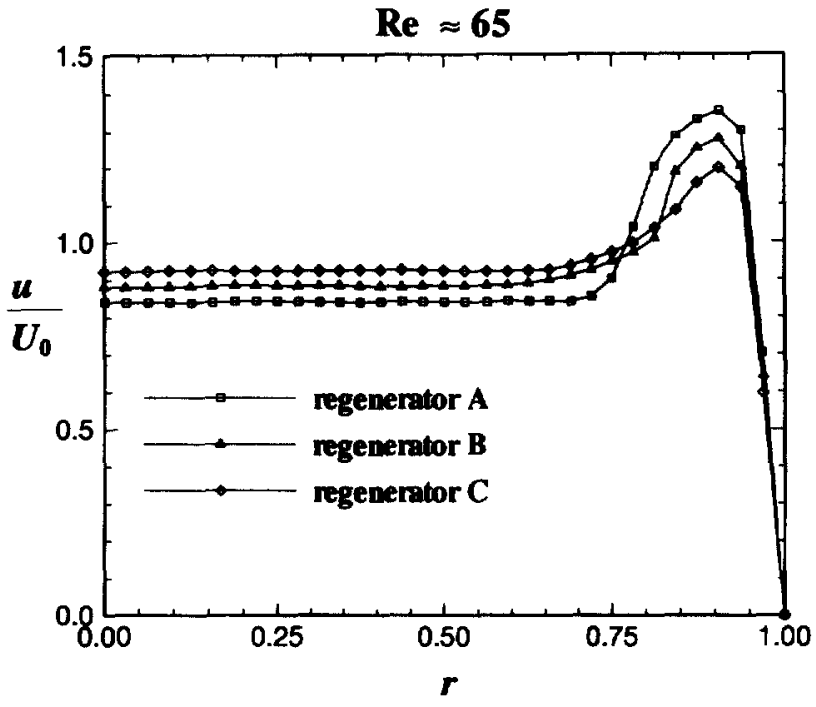

Figure 3 Measured radial velocity profiles for test regenerators $A$, $B$, and $C$ at $R e \approx 65$

is smoother than those for regenerator matrices $\mathrm{A}$ and $\mathrm{B}$. This implies that flow leakage in regenerator $C$, through the contact surface between the matrix and the holding tube, is the least severe of the three test regenerators.

According to the Jones' model, it is necessary to divide the inhomogeneous regenerator matrix into two homogeneous submatrices arranged in parallel. The velocities through both submatrices are assumed to be uniform. This ideal velocity profile through these two submatrices with uniform porosity can be obtained from the measured velocity profile, as shown in Figure 4 for the regenerator matrix $\mathrm{B}$ at $\mathrm{Re} \approx 65$. Note that the $r_{b}$ value is determined from two intersection points between the mean axial velocity and the axial velocity profile. Based on this ideal velocity distribution, the corresponding outer radius for the central submatrix is also illustrated in Figure 4. From the measured velocity profiles at $\mathrm{Re} \approx 65$, Table 2 lists the calculated values of $r_{b}, \phi, \psi, \epsilon_{c}, \epsilon_{b}, h / h_{d}, h_{b} / h_{d}$ and $h / h_{d}$ for all three test regenerators. According to Equation (9), the ratio of each heat transfer coefficient of regenerators $\mathrm{A}, \mathrm{B}$ and $\mathrm{C}$ to that of the well-packed matrix is $0.90,0.94$ and 0.97 ,

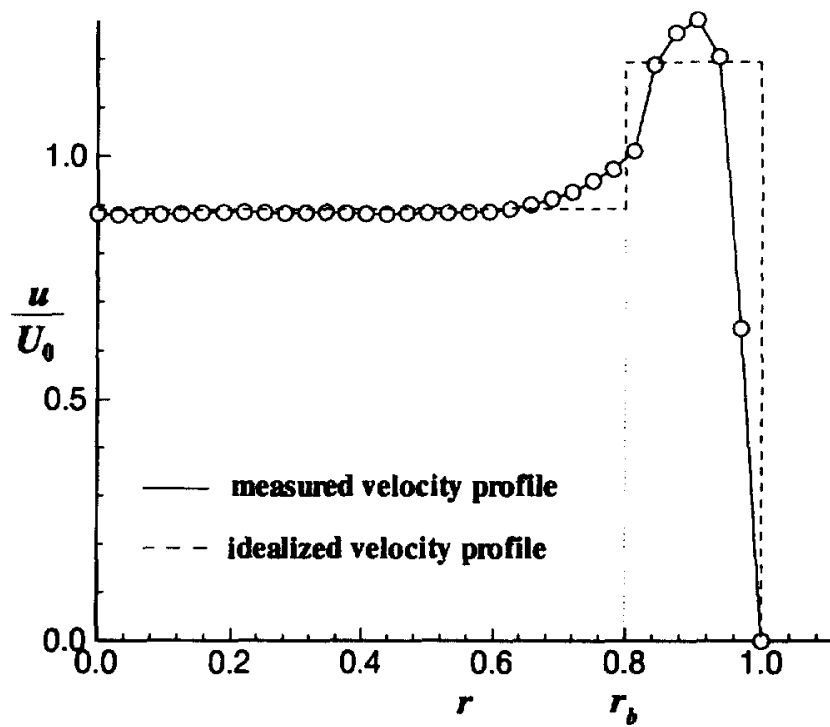

Figure 4 Ideal velocity profile for the regenerator $\mathrm{C}$ through regions of a equivalently homogeneous matrix 
Table 2 Calculated values of $r_{b}, \phi, \psi_{r} \epsilon_{a r} \epsilon_{b u} h_{a} / h_{d r} h_{b} / h_{d}$ and $h / h_{d}$ for test regenerators

\begin{tabular}{|llll|}
\hline Regenerator Type & A & B & C \\
\hline$r_{b}$ & 0.77 & 0.84 & 0.82 \\
$\phi$ & 1.48 & 1.32 & 1.18 \\
$\psi$ & 0.40 & 0.36 & 0.32 \\
$\epsilon_{a}$ & 0.745 & 0.726 & 0.707 \\
$\epsilon_{b}$ & 0.676 & 0.676 & 0.678 \\
$h_{d} / h_{d}$ & 0.89 & 0.93 & 0.97 \\
$h_{b} / h_{d}$ & 0.92 & 0.95 & 0.98 \\
$h / h_{d}$ & 0.90 & 0.94 & 0.97 \\
\hline
\end{tabular}

respectively. Therefore, an increase in the degree of channeling flow may decrease the heat performance of the regenerator matrix. The packing of an oversized wirescreen matrix into the tube is a remedial technique to decrease the flow channeling effect. Velocity profile measurements were conducted at various flow velocities, corresponding to a range of the Reynolds number from 39 . 225. Allowing for experimental error, the variation in calculated values of $r_{b}$ is less than $3.0 \%$ within the range of measured velocity. Thus, only data at $\operatorname{Re} \approx 65$ are used for the evaluation of $\phi$ and $\psi$ values in the present study.

Figure 5 shows the measured Nu values versus the Reynolds number for the three inhomogeneous regenerator matrices investigated compared with those of Hamaguchi et al. 7 It should be noted that the characteristic length in the definition of Reynolds number and Nusselt number in Hamaguchi et al. ${ }^{7}$ is the wire diameter of the wire screen matrix rather than the hydraulic diameter. For comparison, it is necessary to convert their original empirical correlation for the Nusselt number versus Reynolds number to $\mathrm{Nu}=$ $0.614 \mathrm{Re}_{d} 0.56$.

At a similar Reynolds number, the test regenerator with a higher oversize value has a greater Nu value. A value of $C=0.805$ and $n=0.479$ gives the best fit for Equation (5). However, the deviation between the measured data and the predicted value from $\mathrm{Nu}=0.805 \mathrm{Re}^{0.479}$ is beyond the experimental uncertainty. The variation in heat transfer among the test regenerator results from the various degrees

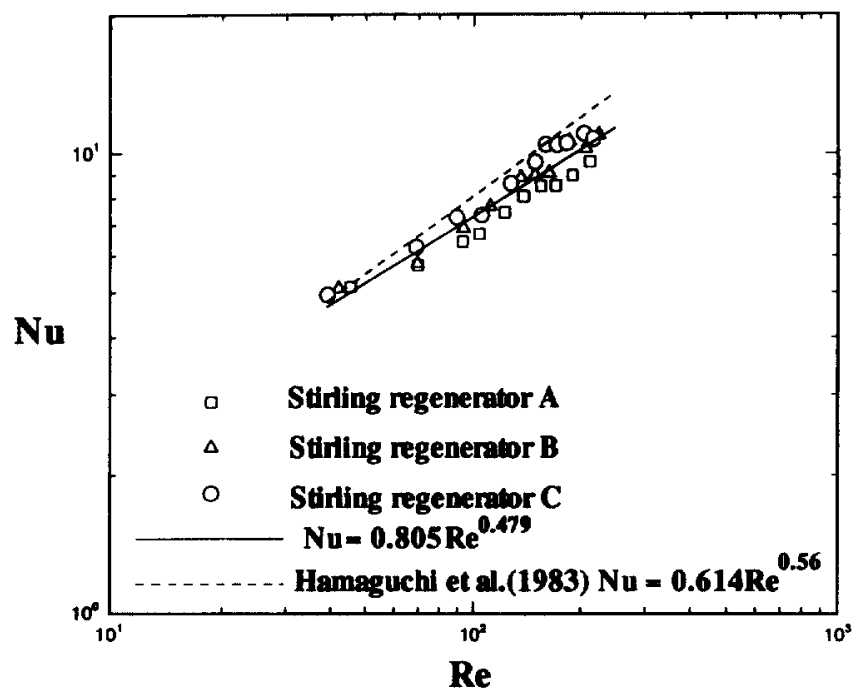

Figure 5 Measured Nu values versus Re for test regenerators $A, B$, and $C$ of flow nonuniformity in the regenerator matrix. Figure 6 shows the predicted values of $\mathrm{Nu}_{d}$ for the perfectly homogeneous matrix from the measured data for various test regenerators. All predicted data of $\mathrm{Nu}_{d}$ closely follow the straight line, expressed by

$$
\mathrm{Nu}_{d}=0.867 \mathrm{Re}^{0.476}
$$

This confirms the analytical model proposed by Jones ${ }^{2}$. Based on the present measured heat transfer results in the regenerator matrices stacked with 560 wire screens, Equation $(10)$ is deduced for the thermal performance of a homogeneous regenerator matrix in a range of Reynolds number from 39-225.

Combining Equation (1), (2), (9), and (10) yields

$$
\begin{aligned}
& \mathrm{Nu}=0.867\left\{\psi\left(\frac{\epsilon_{d}}{\epsilon_{a}}\right)\left(\frac{1-\epsilon_{d}}{1-\epsilon_{a}}\right)^{n-1}\left(\frac{\phi}{(\phi-1) \psi+1}\right)^{n}\right. \\
& \left.+(1-\psi)\left(\frac{1}{(\phi-1) \psi+1}\right)^{n}\right\} \operatorname{Re}^{0.476}
\end{aligned}
$$

which is the general expression for the thermal performance of an inhomogeneous stacked-screen regenerator matrix with flow channeling around the tube periphery. In Equation (11), both values of $\phi$ and $\psi$ are evaluated from the measured radial velocity profile at the regenerator exit. Therefore, Equation (11) shows the effect of flow channeling on the regenerator performance. Also, once the thermal performance of the well-packed regenerator is obtained, the thermal performance of another regenerator

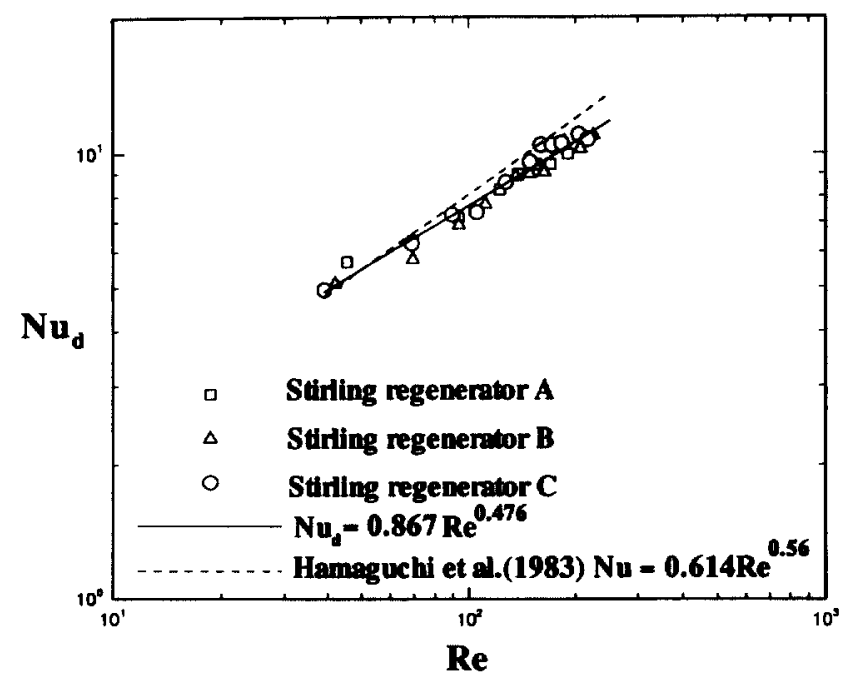

Figure 6 Predicted $\mathrm{Nu}_{d}$ values versus $\mathrm{Re}$ 
with different degree of flow channeling may be easily predicted through Equation (11).

\section{Conclusions}

The flow channeling phenomenon through the regenerator matrix has been verified by exit velocity measurements. The effect of flow channeling on friction loss and heat transfer characteristics of the regenerator matrix have also been experimentally measured. The measured results indicate that the flow channeling effect reduces the thermal performance of a regenerator matrix. The packing of an oversized wire-screen matrix into the tube has been verified as a remedial technique to decrease the flow channeling effect.

A theoretical formula proposed by Jones ${ }^{2}$ to assess the flow channeling effect on the regenerator thermal performance has been modified and applied to the present test Stirling regenerators. The modified formula in Equation (9) shows that the ratios of the heat transfer coefficient of Stirling regenerators $\mathrm{A}, \mathrm{B}$, and $\mathrm{C}$ to those of an equivalently well-packed matrix are $0.90,0.94$, and 0.97 , respectively. Note that the only difference among the three test regenerators is the value of oversize for the regenerator matrix. Equation (11) also serves to predict the thermal performance of any regenerator with different degree of flow channeling.

\section{References}

I. Mickley, H.S., Smith, K.A., and Korchak, E.I., Chemical Engineering Science, (1965) $20237-246$

2. Jones, J.D., ASME J. of Engineering for Gas Turbines and Power, (1989) $111595-600$

3. Chen, P.H., Chang, Zei-Chi, and Huang, B.-J., Cryogenics (1996) 36 , 365-372

4. Liang, C.Y. and Yang, W.J., ASME J. of Heat Transfer (1975) 97, 16-21

5. Chen, P.H. and Chang, Zei-Chi, Int. Comm. Heat Mass Transfer (1996) $2355-68$

6. Kline, S.J. and McClintock, F.A., Mechanical Engineering (1953) 75 , 3-8

7. Hamaguchi, K., Takahachi, S. and Miyabe, H., JSME, Series B (in Japanese) (1983) 49 2001-2010 